\title{
Anodic Oxidation of Acetate and Electroinitiated Polymerization of Acrylic Acid in Aqueous Acetate Solution
}

\author{
Ikuichiro IZUMI*, Akira KunUGi** Tosiyuki SHIMIZU** \\ and Shigeo NAGAURA**
}

\begin{abstract}
Electrode reaction processes in aqueous acetate solution in the presence of acrylic acid were studied at various anodes mainly by means of voltammetry and macroelectrolysis. The electrolysis using a platinum anode gave the oligomer of acrylic acid. Voltammetric investigation suggested that the polymerization of acrylic acid at a platinum anode was electroinitiated by radical intermediates originated from the anodic oxidation of acetate. It has also been shown that the anodic electroinitiated polymerization is a heterogeneous surface reaction occuring on the platinum electrode surface. Similarly, the polymerization of acrylic acid was also observed at a platinum cathode. By considering corresponding cathode potential it was confirmed that the cathodic electroinitiated polymerization was initiated by an atomic hydrogen produced in the course of hydrogen evolution reaction.

On the contrary to the case of the platinum electrode, the electrolysis at both lead dioxide and carbon anodes gave only negligible amounts of the oligomer.
\end{abstract}

\section{Introduction}

Electrochemical production of ethane occurs during electrolysis of aqueous acetate solution. Generally the electrosynthesis of hydrocarbon has been known by the term of Kolbe's reaction. Three principal theories have been put forward for the mechanism of Kolbe's reaction; (1) the discharged ion theory by Brown and Walker ${ }^{1}$, (2) the acylperoxide theory by Fichter $^{2}$ and (3) the hydrogen peroxide theory by Glasstone and Hickling ${ }^{3}$. More recently, the animated investigations of this reaction have been undertaken by many other groups ${ }^{4)}{ }^{(6)}$. Among these reports, it seems that the Brown and Walker mechanism, which states that the reaction proceeds through the following sequence, has been commonly accepted;

$\mathrm{CH}_{3} \mathrm{COO}^{-} \longrightarrow \mathrm{CH}_{3} \mathrm{COO} \cdot+e$

* Department of Chemical Engineering, Nara Technical College (Yamatokoriyama, Nara)

** Department of Applied Chemistry, Faculty of Engineering, Osaka City University (Sugimoto-cho, Osaka)

$$
\longrightarrow \mathrm{CH}_{3} \cdot+\mathrm{CO}_{2}
$$

At a lead dioxide anode ${ }^{7}$, oxygen is evolved with $50 \%$ current efficiency, and no ethane, but carbon dioxide is obtained with a small amount of methanol, formaldehyde etc. At a carbon anode ${ }^{7}$, the current efficiency for the formation of carbon dioxide, methanol and methyl acetate is $97 \%$. Sugino and his coworkers have explained the formation of the above products by radical mechanisms.

Generally, radical formation has frequently been involved in the course of organic electrode processes. Here one might lead to the suggestion that the radicals produced by the electrolysis could be applied as initiators of polymerization reactions. Recently, the attention has been paid to the electroinitiated polymerization as a polymerization reaction under the special circumstance. Studies on the electroinitiated polymerization have been dealt with vinyl monomers such as butadiene ${ }^{8}$, acrylic acid $^{9)}$, methyl methacrylate ${ }^{10)}$, styrene ${ }^{11,212)}$ etc. Beside these, the studies on living polymers of styren $\mathrm{e}^{13)}$ and $\alpha$-methyl styrene ${ }^{14}$ have 
been reported.

Previously the electrode reaction process of nonaqueous solution of acetate in the presence of acrylic acid was studied at a platinum anode by means of macroelectrolysis and voltammetry ${ }^{15)}$. We assumed that the polymerization of acrylic acid was electroinitiated by radical intermediates originated by the anodic oxidation of acetate:

In this paper, the effect of electrode materials (platinum, lead dioxide and carbon) on the polymerization of acrylic acid electroinitiated by Kolbe's reaction in aqueous acetate solution is discussed.

\section{Experimental}

\subsection{Voltammetric measurement}

A platinum wire and a platinum plate were used as a working and a counter electrode, respectively. In the potential sweep voltammetry a usual control circuit was set up by the aid of a Nichia Keiki Model HP-E Potentiostat in connection with a Nichia Keiki Model N-S Special Wave Generator, and the potential was referred to a saturated calomel electrode (S.C.E.). The current-potential curve was recorded by a Toa Electronics Model XYR-1A Recorder.

\subsection{Macroelectrolysis}

A platinum plate $\left(9.8 \mathrm{~cm}^{2}\right)$, a lead dioxide block and a carbon block anodes were used, and a counter electrode was a platinum plate $\left(10.2 \mathrm{~cm}^{2}\right)$. An $\mathrm{H}$-shaped cell with compartment separated by a fritted glass disk was employed. The electrolyte was bubbled with nitrogen gas and kept in the region of $-5 \sim$ $+5^{\circ} \mathrm{C}$. A Yanagimoto Model VE-3 Controlled Potential Electrolyser was employed as an electric source. Polymerization products were precipitated with a large quantity of methanol, redissolved in water, reprecipitated, dried and weighed.

\subsection{Reagents}

Acrylic acid of a special reagent-grade was distilled under reduced pressure and a middle cut, bp $39 \sim 40^{\circ} \mathrm{C} / 10 \mathrm{mmHg}$, was used. All the other chemicals were of a special reagentgrade and were used without further purification.

\section{Results and Discussion}

\subsection{Anodic reaction at a platinum anode}

3.1.1 Votammetric behaviour The current-potential curve for an aqueous acetate $(\mathrm{pH} 3.91)$ at a platinum anode is shown as curve (a) in Fig. 1, in which an anodic current peak was observed at $c a .1 .95 \mathrm{~V}$ vs. S.C.E. As is evident from Fig. 2, in which the effect of concentration of acetate is represented, anodic current decreased with increasing concentration of acetate in lower potential region than the peak potential. As already reported ${ }^{16}$, anodic current in lower and higher potential region than the peak potential may be due to the discharge of water (or hydroxy ion) and of acetate (that is Kolbe's reaction), respectively. And also the suppressing effect of acetate on oxygen evolution reaction was assumed to be caused by the decrease of effective platinum surface area by adsorption of acetate anion on the platinum anode.

As is evident from curve (b) in Fig. 1, the current-potential curves for an aqueous acrylic acid solution give no current peak. However, in aqueous acrylic acid solution containing acetate, a current peak was observed on the current-potential curve and the corresponding peak potential was shifted to more positive

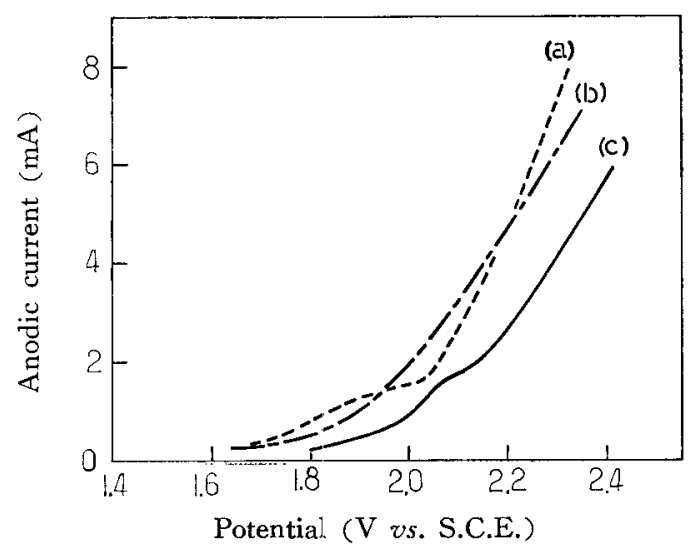

Fig. 1 Current-potential curves measured in aqueous potassium hydroxide solutions of $\mathrm{pH} 3.91$ at a platinum electrode

(a): with $1.3 M$ acetic acid, (b): with $0.93 M$ acrylic acid, (c); with $1.3 M$ acetic acid and $0.93 M$ acrylic acid 


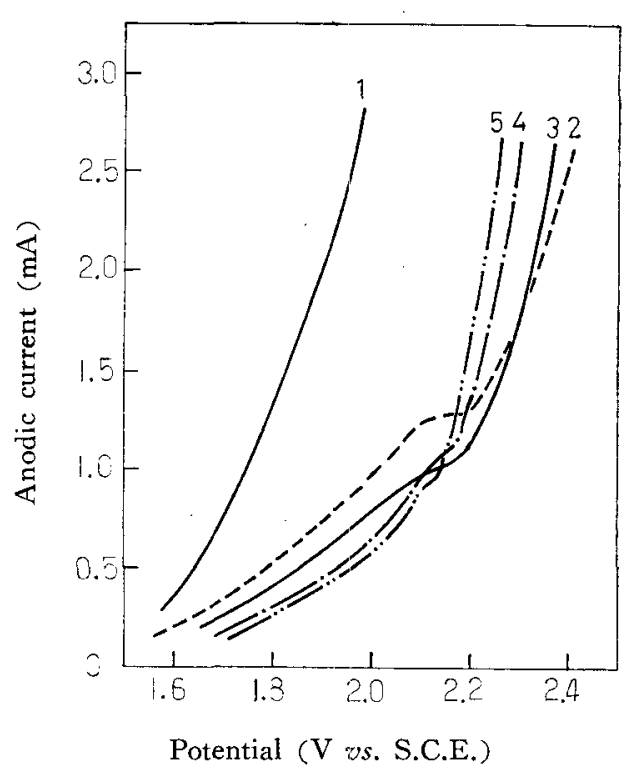

Fig. 2 Effect of the concentration of acetate on current-potential curve in phosphate buffer solutions of $\mathrm{pH} 6.0$

Concn. of potassium acetate: (1) $O M$, (2) $0.3 M$, (3) $0.45 M$, (4) $0.6 M$, (5) $1.2 M$

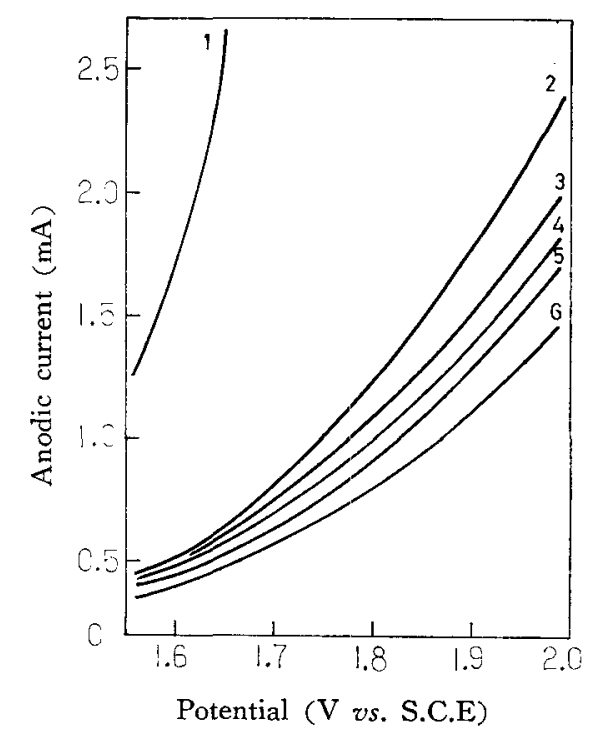

Fig. 3 Effect of the concentration of acrylic acid on current-potential curve in phosphate buffer solutions of $\mathrm{pH} 6.0$

Concn. of acrylic acid: (1) $0 M$, $0.32 M$, (3) $0.81 M$, (4) $0.93 M$, $1.91 M, \quad$ (6) $2.41 M$ potential by the addition of acrylic acid ${ }^{* 1}$ as is shown in Fig. 1. Furthermore, we should point out that the current for oxygen evolution reaction decreased by the addition of acrylic acid as is shown in Fig. 3. Assuming that the decrease of the current is caused by the blocking of the electrode surface by the adsorption of acrylic acid, the surface coverage of acrylic acid $(\theta)$ was evaluated by the following equation, whose treatment has been reported in the previous paper $^{18)}$,

$$
\theta=\left[\left(i_{0}-i\right) / i_{0}\right]_{E=\text { const }}
$$

where $i_{0}$ and $i$ are the anodic current when acrylic acid is absent and when the concentration of acrylic acid is $C(M)$, respectively. The calculated coverage of acrylic acid is plotted $v s$. the logarithmic concentration of the acid in Fig. 4. The curves indicate that the adsorption of acrylic acid obeys Temkin isotherm, $\theta=k \ln C+b^{* 2}$, and the coverage increases with the anode potential arriving a saturated coverage.

Thus it is suggested that acetate anion and acrylic acid are co-adsorbed on the electrode surface, and that the former discharges in higher potential region than the potential

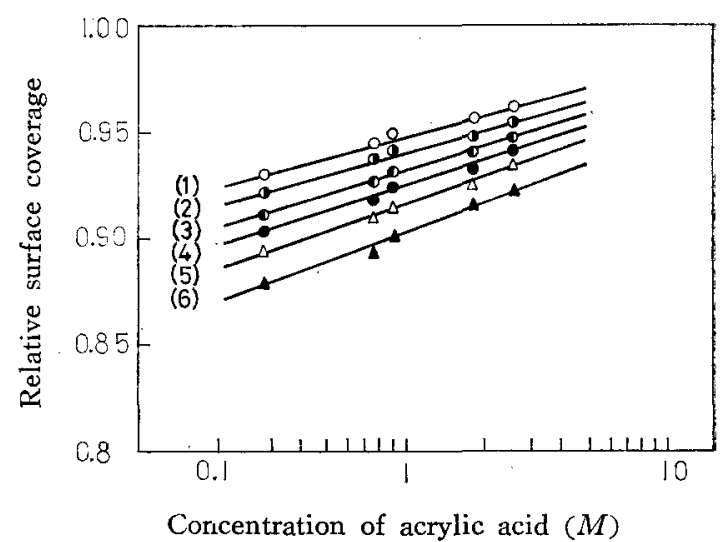

Fig. 4 Relation between the relative surface coverage and the concentration of acrylic acid

Potential: (1) $1.95 \mathrm{~V}, 2.00 \mathrm{~V},(2) 1.90 \mathrm{~V}$, (3) $1.85 \mathrm{~V}$, (4) $1.80 \mathrm{~V}$, (5) $1.75 \mathrm{~V}$, (6) $1.70 \mathrm{~V}$

\footnotetext{
*1 The shift of anode potential has been frequently found if other organic substrates were used in place of acrylic acid ${ }^{17)}$. What has caused this shift? We could not clear up the question.

*2 Both $k$ and $b$ are constants in the equation.
} 
corresponding to current peak (or rather plateau).

3.1.2 Polymerization at a platinum anode From the voltammetric results, the main anodic reaction for the aqueous solution containing both acrylic acid and acetate is assumed not to be the discharge of acrylic acid but of acetate in the region of $1.9-2.4 \mathrm{~V}$ vs. S.C.E. If the discharge of acetate involves radical intermediates such as acetoxy and methyl radicals followed by its decarboxylation, the polymerization of acrylic acid will be initiated by electrolysis of aqueous acetate solutions containing acrylic acid. As is shown as curve (a) in Fig. 5, the anodic polymerization commences to occur at $1.9 \mathrm{~V}$, at which acetate discharges ${ }^{19)}$, and then the oligomer yield rapidly increases at more positive potential than $c a .2 .15 \mathrm{~V}$. Anode potential for the rapid increase of the oligomer yield is in agreement with the peak potential exhibited in the current-potential curve for aqueous acetate solution in the presence of acrylic acid (see curve (c) in Fig. 1).

The relation between the concentration of acetate and oligomer yield was examined by electrolysis at $2.2 \mathrm{~V}$, which is shown in Fig. 6. A characteristic behaviour was observed in Cathode potential ( $\mathrm{V}$ vs. S.C.E.)

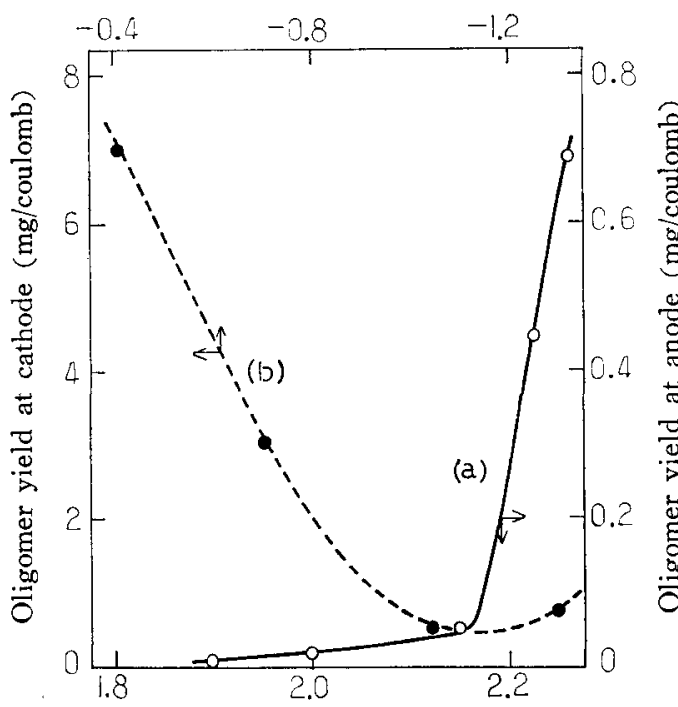

Anode potential (V vs. S.C.E.)

Fig. 5 Potential dependence of oligomer yield at platinum electrodes in $2 N$-KOH with $2.5 M$ acrylic acid and $4.1 M$ acetic acid

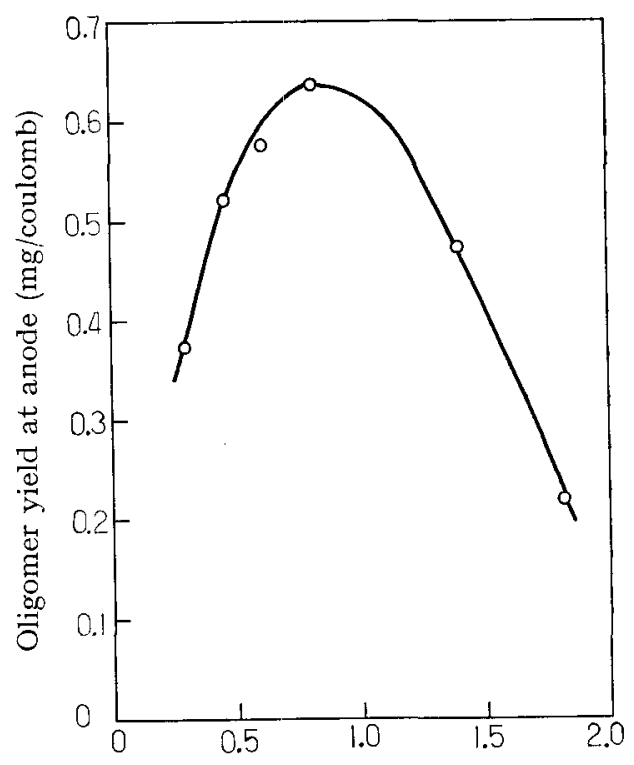

Concentration of potassium acetate $(M)$

Fig. 6 Oligomer yield as a function of the concentration of potassium acetate

Concentration of acrylic acid: $2 M$

Electrolysis current: $90 \mathrm{~mA}$

the relation. Namely, the oligomer yield increased with the concentration of acetate in its lower concentration range, and at a limited value of the concentration the yield begins to decrease. A decreasing reaction velocity with concentration of acetate cannot be understood by any homogeneous reaction kinetics, but can be explained by quoting the knowledge for gas phase as follows. When the polymerization (really, oligomerization) ${ }^{* 3}$ is initiated at a solid electrode surface, the rate equation can be expressed as

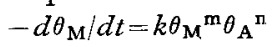

where $\theta_{\mathrm{M}}$ and $\theta_{\mathrm{A}}$ are the fractions of electrode surface covered by acrylic acid and acetate, respectively, and then $m$ and $n$ are the reaction order with respect to each species. The other symbols have their usual significance. If coadsorption of acrylic acid and acetate obeys the Langmuir isotherm*4,

*s We inclusively used the term "polymerization" in place of the term "oligomerization".

*t It has already been reported that the adsorption of acetate obeys the Langmuir isotherm ${ }^{(8)}$. While the adsorption of acrylic acid in the absence of acetate obeys the Tomkin type isotherm, the Langmuir isotherm can be used for the adsorption of acrylic acid in the presence of acetate. Therefore, we can explain the present phenomena without difficulty. 
$\theta_{\mathrm{j}}=b_{\mathrm{j}} c_{\mathrm{j}} /\left(1+b_{\mathrm{j}} c_{\mathrm{j}}\right)$,

(4)

where $c_{\mathrm{j}}$ means the bulk concentration of component $j$ and $b$ is a constant.

Introducing equation (4) into equation (3), we obtain

$$
\begin{aligned}
-d \theta_{\mathrm{M}} / d t & =k\left(b_{\mathrm{M}} c_{\mathbf{M}}\right)^{\mathrm{m}}\left(b_{\mathrm{A}} c_{\mathbf{A}}\right)^{\mathbf{n}} /(1 \\
& \left.+b_{\mathbf{M}} c_{\mathbf{M}}+b_{\mathbf{A}} c_{\mathbf{A}}\right)^{\mathbf{m}+\mathbf{n}} .
\end{aligned}
$$

If the reaction orders with respect to both acrylic acid and acetate are unity in the present system and then the rate is proportional to the oligomer yield $(Y, \mathrm{~g} /$ coulomb), conversion of acrylic acid during $\Delta t(\mathrm{sec})$ can be written as

$Y=k\left(b_{\mathrm{M}} c_{\mathrm{M}}\right)\left(b_{\mathrm{A}} c_{\mathrm{A}}\right) /\left(1+b_{\mathrm{M}} c_{\mathrm{M}}+b_{\mathrm{A}} c_{\mathrm{A}}\right)^{2}$

When the concentration of acrylic acid is constant, the oligomer yield changes with the concentration of acetate giving a maximum, at which $c_{\mathrm{A}}$ is $\left(1-b_{\mathrm{M}} c_{\mathrm{M}}\right) / b_{\mathrm{A}}$. From the results in Fig. 6 and the treatment considered above, we may conclude that the electroinitiated polymerization reaction proceeds at the electrode surface and not homogeneously in the solution, as described in the voltammetric behaviour.

The free radical character of the electroinitiated polymerization was supported by our test with free radical inhibitors. With benzoquinone $(0.12 M)$ in the anode compartment, no anodic oligomer was given. From this inhibition effect on growth process, the likelihood of radical mechanism at anode is suggested. Therefore, it may be concluded that radical intermediates involved in the discharge of acetate at anode also take part in the initiation step of polymerization of acrylic acid*5.

\subsection{Cathodic reaction at a platinum cathode}

Oligomer was also obtained in a cathode compartment. As shown in Fig. 5 (curve (b)), the oligomer yield decreased more and more at less positive cathode potential. It was shown from the following results that the polymerization at a cathode was initiated by a hydrogen atom produced in the course of cathodic reduction of proton; (1) hydrogen evolution reaction occurred in the region of potential examined but acrylic acid itself was not reduced electrochemically, (2) The oligomer yield

*s Electrolysis of acrylic acid in aqueous potassium hydroxide solution without acetate gave oxygen as a main product, a small amount of acetylene and no oligomer. at a cathode by macroelectrolysis without acetate was nearly equivalent to with acetate.

In order to determine whether the polymerization at a platinum cathode propagates with radical mechanism or not, use was made of p-hydroquinone as radical inhibitor and the procedure was essentially similar to the experiment at an anode. The oligomer yield at a cathode decreased by approximately $91 \%$ by the addition of radical inhibitor $(0.14 M)$.

Consequently, the cathodic polymerization was assumed to be initiated by a hydrogen atom involved in the discharge process of proton and to be propagated with radical mechanism.

\subsection{Analysis of oligomer at a platinum electrode}

Intrinsic viscosity was measured in $2 \mathrm{~N}$ $\mathrm{KOH}$ aqueous solution at $25^{\circ} \mathrm{C}$ in Ostwald viscometers, and the molecular weight was determined from the Staudinger equation for a region of lower molecular weight, $\eta_{\mathrm{sp}}=$ $K_{\mathrm{m}} M\left(K_{\mathrm{m}}=2.9 \times 10^{-4}\right)^{20}$. An example of the results is shown in Table 1. The elementary analysis of the oligomer obtained at an anode was the following; C, $41.33, \mathrm{H}, 5.25,0,53.42 \%$. In the infrared absorption of the oligomer at an anode, the characteristic absorption bands arising from the carboxyl groups were observed with a weak absorption due to hydrogen bonding. Further analysis for the structure of the oligomer was not carried out.

Table 1 Viscosity measurement of oligomer

Electrolyte: $2.5 M$ acrylic acid $-4.1 M$ acetic acid- $1 N-\mathrm{KOH}$ solution

Electrolysis current: $75 \mathrm{~mA}$

\begin{tabular}{l|c|c|c}
\hline \hline & $\begin{array}{l}\text { Oligomer yield } \\
\text { (mg/coulomb) }\end{array}$ & $\eta_{\mathbf{s p}}$ & $\begin{array}{l}\text { Molecular } \\
\text { weight }\end{array}$ \\
\hline $\begin{array}{l}\text { Oligomer } \\
\text { at cathode }\end{array}$ & 0.6827 & 3.15 & 1090 \\
\hline $\begin{array}{l}\text { Oligomer } \\
\text { at anode }\end{array}$ & 0.3475 & 0.13 & 450 \\
\hline
\end{tabular}

\subsection{Polymerization at a lead dioxide and a carbon anodes}

Koehl $^{213}$ reported that electrolysis of simple aliphatic acids in aqueous and anhydrous solutions at an anode gave high yield of products, for the production of which carbo- 
nium ion like species are reaction intermediates. For example, from acetate in aqueous and :anhydrous acetic acid, methyl acetate was formed in $82 \%$ and $56 \%$ yields, respectively. At a lead dioxide ${ }^{7}$, anodic oxidation of aqueous acetate solution produced oxygen, carbon dioxide, formaldehyde and methanol, and then Sugino and his co-workers have explained the formation of above products by the hypothesis that radical intermediates are involved in such an anodic oxidation. We presented in the previous paper ${ }^{6)}$ that one electron was involved in an anodic oxidation of acetate in acetonitrile at a platinum, a carbon and a lead dioxide anodes, and then anodic oxidations proceeded through a free radical sequence irrespective of nature of an electrode material.

If radical intermediates take part in the anodic oxidation of acetate in an aqueous solution at a carbon and a lead dioxide anodes, the resulted radical species may be applied to electroinitiated polymerization. In this point, a test whether oligomer is formed or not was examined by electrolysis of an aqueous acetate solution in the presence of acrylic acid at these anodes. The formation of the oligomer of acrylic acid at the carbon anode was significantly reduced as compared with that at the platinum anode, and the yield was a trace of amount. Similarly, the oligomer obtained at the lead dioxide anode was a negligible amount. The poor formation of oligomer was observed from turbidity of the electrolyte with a large amount of methanol.

Thus it is plausible to assume that the intermediates during the anodic oxidation of acetate in an aqueous solution at the carbon and the lead dioxide anodes are not radical species but carbonium ion like species, as supposed by Koehl, though the difficulty of polymerization is understood to be attributed to the preferential oxygen evolution at these electrodes.

As described above, attempts to polymerize acrylic acid at the carbon and the lead dioxide anodes were unsuccesful, and therefore the investigation of electroinitiated polymerization of acrylic acid at these electrodes was not undertaken furthermore.

\section{Conclusion}

The main conclusions obtained in this work can be summarized as follows.

1) Voltammetric behaviour suggested that acetate and acrylic acid were co-adsorbed on a platinum electrode, and that anodic current in higher potential region than $1.9 \mathrm{~V}$ vs. S.C.E. might be due to the discharge of acetate.

2) From preparative results of macroelectrolysis, it was found that the polymerization of acrylic acid was electroinitiated at a platinum electrode surface by radical intermediates originated from the discharge of acetate.

3) The polymerization of acrylic acid at a platinum cathode might be initiated by a hydrogen atom involved during the discharge of proton and propagated with a radical character.

4) Products by the anodic and the cathodic polymerization of acrylic acid at platinum electrodes were oligomers of low molecular weight.

5) At a carbon and a lead dioxide anodes, the oligomer yield in an anode compartment was a trace of amount. From the fact, it might be considered that the intermediates during the anodic oxidation of acetate at these electrodes were not radical species but carbonium ion like species, though the difficulty of polymerization was understood to be caused by inhibition of oxygen evolved at these electrodes.

$$
<\mathrm{E} 425>\text { (Received May 23, 1975) }
$$

\section{References:}

1) A.Brown, J. Walker, Ann. 261, 107 (1891).

2) F. Fichter, Trans. Amer. Electrochem. Soc. 45, 131 (1924).

3) S. Glasstone, A. Hickling, J. Chem. Soc. 1878 (1934).

4) B.E. Conway, H. Dzriciuch, Canad. J. Chem. 41, 21 (1963).

5) L. Eberson, Acta Chem. Scand. 17, 2004 (1963).

6) A. Kunugi, H. Urata, S. Nagaura, This Journal 36, 237 (1968).

7) N. Sato, T. Sekine, K. Sugino, ibid. 34, 119(1966).

8) W.B. Smith, H.G. Gilde, J. Amer. Chem. Soc. 83, 1355 (1961).

9) W.B. Smith, D.T. Manning, J. Polymer Sci. 59, S 45 (1962).

10) B.L. Funt, K.C. Yu, ibid. 62, 359 (1962).

11) B.L. Funt, S.N. Bhadani, Canad. J. Chem. 42, 2733 (1964).

12) B.L. Funt, S.W. Lauurent, ibid. 42, 2728 (1964).

13) B.L. Funt, D. Richardson, ibid. 44, 711 (1966). 
14) N. Yamazaki, S. Nakahama, S. Kambara, Polymer Letters 3, 57 (1965).

15) I. Izumi, A. Kunugi, S. Nagaura, Nippon Kagaku Zasshi 91, 1128 (1970).

16) A. Kunugi, S. Iseki, K. Ohashi, S. Nagaura, This Journal_37, 41 (1969).

17) H. Nagamori, T. Sekine, Abstract of the 35 th Annual Meeting of the Electrochemcal Society of
Japan, (1968), p. A-69 Toyama.

18) K. Sasaki, I. lzumi, K. Ohashi, T. Uemura, S. Nagaura, Nippon Kagaku Zasshi 87, 918 (1966).

19) D.A. Fairweather, O.J. Walker, J. Chem. Soc. 3116 (1926).

20) H. Staudinger, E. Tromdorff, Ann. 502, 201(1933).

21) W.J. Koehl, Jr., J. Amer. Chem. Soc. 86, 4686; (1964). 